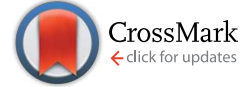

Cite this: RSC Adv., 2015, 5, 106971

Received 14th October 2015 Accepted 4th December 2015

DOI: $10.1039 / c 5 r a 21345 b$

www.rsc.org/advances

\section{Novel nitrogen-rich energetic macromolecules based on 3,6-dihydrazinyl-1,2,4,5-tetrazine $\uparrow$}

\author{
Adva Cohen, Qi-Long Yan, Avital Shlomovich, Alexander Aizikovich, Natan Petrutik \\ and Michael Gozin*
}

Nitrogen-rich energetic macromolecules were synthesized by reacting dihydrazinyl-1,2,4,5-tetrazine $(\mathrm{DHTz})$ with various diisocyanates. It has been shown that polymerization or cyclization reactions could take place depending on solvent systems and reaction conditions. The formed macrocycles and polymers were comprehensively characterized by IR, NMR, high resolution MS and SEM, while the thermal stability was studied by DSC. The energetic properties of our new compounds were investigated by bomb calorimetry, along with calculations using the EXPLO-5 code. It was found that macrocycle CDHTZ-TM and corresponding polymer P-DHTZ-TM prepared on a basis of DHTZ and tetramethylene diisocyanate have larger heats of combustion than RDX, GAP and polyGLYN. C-DHTZ-TM has a comparable density and larger detonation velocity than TNT. The nitrogen content of C-DHTZ-TM and P-DHTZ-TM is larger than 47\%. The thermal stability of DHTz-based energetic macromolecules was found to be higher than currently used energetic polymers including GAP, polyNIMMO and polyGLYN. The ADN based compositions bonded by $60 \%$ (by weight) of P-DHTZ-HM and P-DHTZ-MB polymers have higher theoretical specific impulse than that bonded by polyGLYN. Therefore, our new compounds could be promising ingredients as energetic binders or fillers. Moreover, all of the macrocycles have potential to be used as energetic gelators in gel propellants.

\section{Introduction}

In the development of high performance energetic compositions, one of the promising ways could be replacement of the current inert binders (e.g. hydroxyl-terminated polybutadiene "НTPB" and carboxyl-terminated polybutadiene "СТРВ") with energetic polymers. ${ }^{1}$ One of the basic requirements of the newly developed energetic binders is to be compatible with ecofriendly high energetic oxidizers, such as ammonium nitrate (AN), ammonium dinitramide (ADN), hydrazinium nitroformate (HNF) and cyclotetra-methylene tetranitramine. In past decades, several new energetic binders have been developed for solid propellants, including azide functional polymers and nitrato polyether. For example, the former group includes glycidyl azide polymer (GAP), ${ }^{2,3}$ poly-3,3-bis(3-azidomethyl)oxetane (polyBAMO) ${ }^{4}$ and poly-(3-azidomethyl)-3-methyloxetane (polyAMMO). ${ }^{5}$ The latter group comprises poly-3-nitratomethyl-3methyloxetane (polyNMMO), ${ }^{6}$ copolymer of BAMO/NMMO, ${ }^{7}$ poly-glycidylnitrate (polyGLYN), ${ }^{8}$ polyvinyl nitrates (PVN), ${ }^{9}$ and nitrated polybutadienes (NPB). ${ }^{10}$ However, there are still several problems need to be solved for abovementioned energetic

School of Chemistry, Faculty of Exact Science, Tel Aviv University, Tel Aviv, 69978, Israel. E-mail: cogozin@gmail.com; Tel: +972-36405878

$\dagger$ Electronic supplementary information (ESI) available: The preparation methods, HRMS spectra, NMR spectra and the molecular structure of macrocycles in different sizes. See DOI: 10.1039/c5ra21345b polymers before they could be more widely applied. For instance, the propellants containing GAP polymer usually have weak mechanical strength, due to the presence of voids caused by gas formation during curing process with isocyanates. ${ }^{11}$ Therefore, new attempts have been made to transform GAP from a thermoset polymer to a thermoplastic elastomer, ${ }^{12}$ by binding functional moieties, including chain extenders 1,4butanediol and $N$-(2-cyano-ethyl) diethanolamine. ${ }^{13}$ Alternatively, GAP can be copolymerized with BAMO to form new bifunctional polymers with improved mechanical strength. ${ }^{14}$

Except for the mechanical strength, some other important issues also need to be considered, including the sensitivity, oxygen balance (nitrogen content), as well as energy content. ${ }^{15}$ The currently available energetic polymers are, in most cases, based on branched azido or nitrate groups, which are not insensitive with relatively small energy and nitrogen content $(<43 \%)$. For instance, GAP has nitrogen content of about $42 \%$, while PVN contains $13-16 \%$ of nitrogen, which is comparable to nitrocellulose. ${ }^{9}$ In addition to nitrate and azido groups, the picryl groups have also been introduced as branches of linear poly(ethylenimine) and poly(vinyl-amine), to form energetic polymers. ${ }^{16}$ In order to improve the safety of azido-polymers, a new energetic polymer - poly(vinyl acetate azide) (PVAA), was synthesized via a two-step process involving initial chloroacetylation with subsequent azidation of poly(vinyl alcohol). ${ }^{17}$ The strategy in this case was to replace the azido and nitrate 
groups with safer energetic side-groups, such as triazole, tetrazole, triazine and tetrazine, which also improved the nitrogen contents in these polymers. ${ }^{\mathbf{1 8 - 2 0}}$ In fact, the synthesis of polymers based on triazole and triazine moieties could be traced back to $1950 \mathrm{~s}^{21-23}$ The tetrazole and tetrazine could be even better moieties for constructing energetic polymers due to their higher nitrogen content. ${ }^{24-26}$ It has been shown that the tetrazole- and tetrazine-based energetic polymers are usually more thermally stable and less sensitive. Therefore, such nitrogenrich polymers could be very promising candidates for energetic applications. For example, poly(5-vinyltetrazole) is one of the most attractive polymers containing tetrazoles, which decomposes at around $260{ }^{\circ} \mathrm{C} .{ }^{27}$

However, almost all of the abovementioned energetic polymers were prepared via replacing some of monomer functional groups with energetic groups, which also may cause thermal stability and sensitivity problems. To address these issues, here we report the preparation and characterization of novel energetic cyclic macromolecules and polymers, based on 3,6dihydrazinyl-1,2,4,5-tetrazine (DHTz) monomer, ${ }^{28-30}$ as an inbackbone building block.

\section{Results and discussion}

\subsection{Selectivity in cyclization $v s$. polymerization}

The macromolecules DHTZ-HM, DHTZ-TM and DHTZ-MB were synthesized by reacting DHTz with various bi-substituted isocyanates in polar aprotic solvents at $20{ }^{\circ} \mathrm{C}$ for $48 \mathrm{~h}$ (Scheme 1). DHTZ-HM was prepared from hexamethylene diisocyanate,
DHTZ-TM from tetramethylene diisocyanate and DHTZ-MB from toluene diisocyanate, respectively.

Formation of macrocyclic vs. polymeric structures could be directed by using appropriate reaction conditions, in terms of reaction solvent, dilution and temperature regime. Different conditions were utilized to synthesize the DHT based macrocyclic compounds: temperature, solvent types and solvent amounts. It has been reported that the size of the macrocycles is temperature dependant. ${ }^{31}$ The most appropriate temperature for preparation of all macromolecules was found to be $20{ }^{\circ} \mathrm{C}$ (Table S1, ESI $\dagger$ ). In addition to temperature, the effects of solvent types and volumes were on the cyclization and polymerization were investigated (Table S2, ESI $\dagger$ ). Our initial investigations related to polymerization and cyclization of DHTz precursor showed mostly formation of mixed macrocycles with different sizes. Extensive evaluation of various experimental conditions leads us to two optimized methods (designated as A and B in Scheme 1). In case of C-DHTZ-HM and CDHTZ-MB, method A (based on use of DMF/THF mixture as a solvent) allowed preparation of practically pure cyclic macromolecule with $n=2$ in (meaning that each macrocycle contains just two repeating units; Scheme S1, ESI $\dagger$ ). Yet, method A was found unsuitable for preparation of C-DHTZ-TM macrocycle, since it leads to precipitation of a mixture of different size macrocycles ( $n=1,2,3$ etc.). To overcome this problem, we developed method B, which was based on use of DMF as a solvent.

Significant efforts were also invested in our attempts to develop viable methodologies for size-specific preparation of

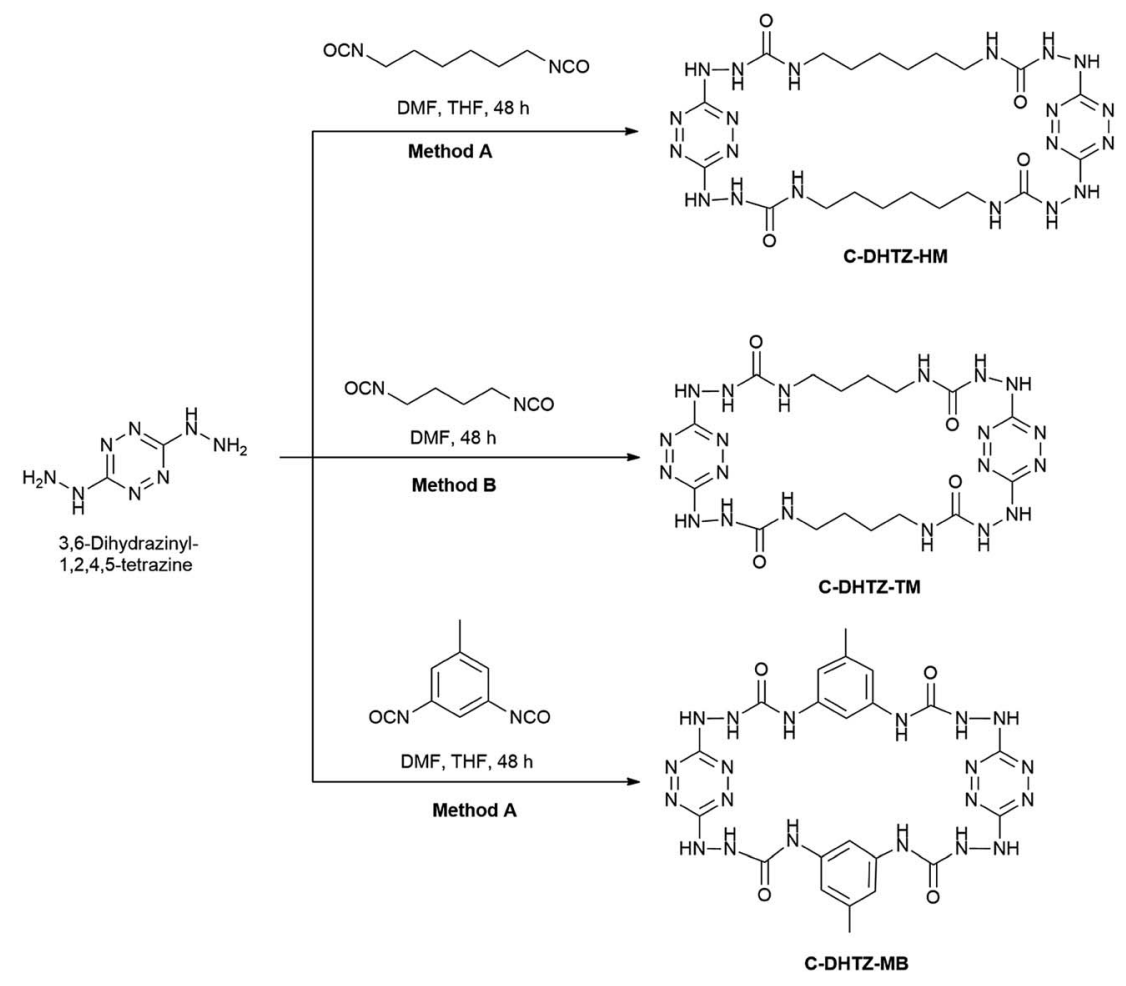

Scheme 1 Synthetic routes for C-DHTZ-HM, C-DHTZ-TM and C-DHTZ-MB macrocycles. 
various DHTz-based macrocycles with $n=3$ or $n=4$ (Scheme S1, ESI†). However, based on LC-MS analysis, only mixtures of macrocycles were obtained in relatively low yields. The major conclusion of these studies was that for size-specific formation of macrocycles with $n>2$ would require the use of template ligands.

We found that under certain reaction conditions, instead of cyclic macromolecules, polymeric P-DHTZ-HM, P-DHTZ-TM and P-DHTZ-MB could be obtained (Scheme 2). In order to promote polymerization reaction and to avoid formation of macrocycles, the amount of used solvent should be reduced to a minimum. We found that all three polymerization reactions could be carried out under the same experimental conditions, using DMF as a solvent. In contrast to macrocycles that precipitated in form of amorphous powder, the polymers precipitated in form of amorphous rigid blocks. Macrocycles $v s$. polymers also showed a significant difference in their solubility, where the macrocycles were somewhat soluble in DMF and DMSO, while corresponding polymers were practically insoluble in all tested organic solvents. The solubility of the macrocycles in DMF and DMSO are shown in Table S4 (ESI†).

\subsection{Molecular structure and stoichiometry}

The elemental compositions and molecular structures of the obtained macrocycles were obtained on a basis of elemental analysis, FTIR, ESI-MS, ${ }^{1} \mathrm{H}$ and ${ }^{13} \mathrm{C}$ NMR and high resolution MS (HRMS) techniques (Fig. S1-S11, ESI $\dagger$ ).

2.2.1 FTIR analysis. Comparison of FTIR spectra of pure macrocycles $(n=2)$ and the corresponding polymers, allows to observe significant similarity between compounds comprised of the same building blocks (Fig. 1) and the corresponding peaks are identified in Table 1.

It was found that frequencies of the chemical bonds in the polymer are generally somewhat lower than the same bonds in the macrocycles. The DHTZ-HM has the longest alkane chain, resulting in higher intensity peaks at this $\mathrm{C}-\mathrm{H}$ region. The peak at $3221 \mathrm{~cm}^{-1}$ was attributed to aromatic $\mathrm{C}-\mathrm{H}$ stretch of tolyl unit in DHTZ-MB. The peaks in the range of $1662-1631 \mathrm{~cm}^{-1}$ could be attributed to urea's carbonyl groups, where peaks at 1639$1631 \mathrm{~cm}^{-1}$ can be attributed to the $\mathrm{N}-\mathrm{H}$ bending of amine groups. The peaks at $1460-1440 \mathrm{~cm}^{-1}$ correspond to the $\mathrm{C}-\mathrm{H}$ bend of alkane chains and peaks in $1250-1020 \mathrm{~cm}^{-1}$ region correspond to $\mathrm{C}-\mathrm{N}$ bond stretch of these chains. Interestingly, it is possible that peaks at $2346-2361 \mathrm{~cm}^{-1}$ in the macrocycles correspond to the stretching or bending of the entire macrocyclic ring structure. For example, C-DHTZ-TM has the smallest ring size, resulting in two sharp peaks at $2361 \mathrm{~cm}^{-1}$. A smaller peak at the latter region could also be seen in a spectrum of $\mathbf{P}$ DHTZ-TM, suggesting presence of C-DHTZ-TM as an impurity (this conclusion received an additional support from SEM analysis of the P-DHTZ-TM).

2.2.2 Elemental analysis. Due to insolubility of DHTZcontaining polymers, in addition to FTIR, analysis of their chemical composition was also based on elemental analysis. Despite all our attempts to remove DMF residue (reaction solvent) from solid polymers by thorough extraction with water, followed by keeping treated polymers under vacuum $(<100$ mbar) for several days, the elemental analysis of these materials still showed presence of DMF (and water). The amount of the polymer-entrapped DMF was in the range of $10.5 \%$ to $31.7 \%$, while the entrapped-water content reached up to $3.5 \%$ (Table 2). The elemental analysis also indicates that there were no detectable amounts of entrapped starting isocyanates, their hydrolysis products and corresponding ureas. Similar results were previously reported by Klapötke for tetrazolyl hydrazinebased polymers, which had entrapped DMF content in the range of $7.5 \%$ to $17.3 \%$ (and entrapped water up to $2.7 \%$ ). ${ }^{23} \mathrm{In}$ case of macrocycles, the elemental analysis also showed

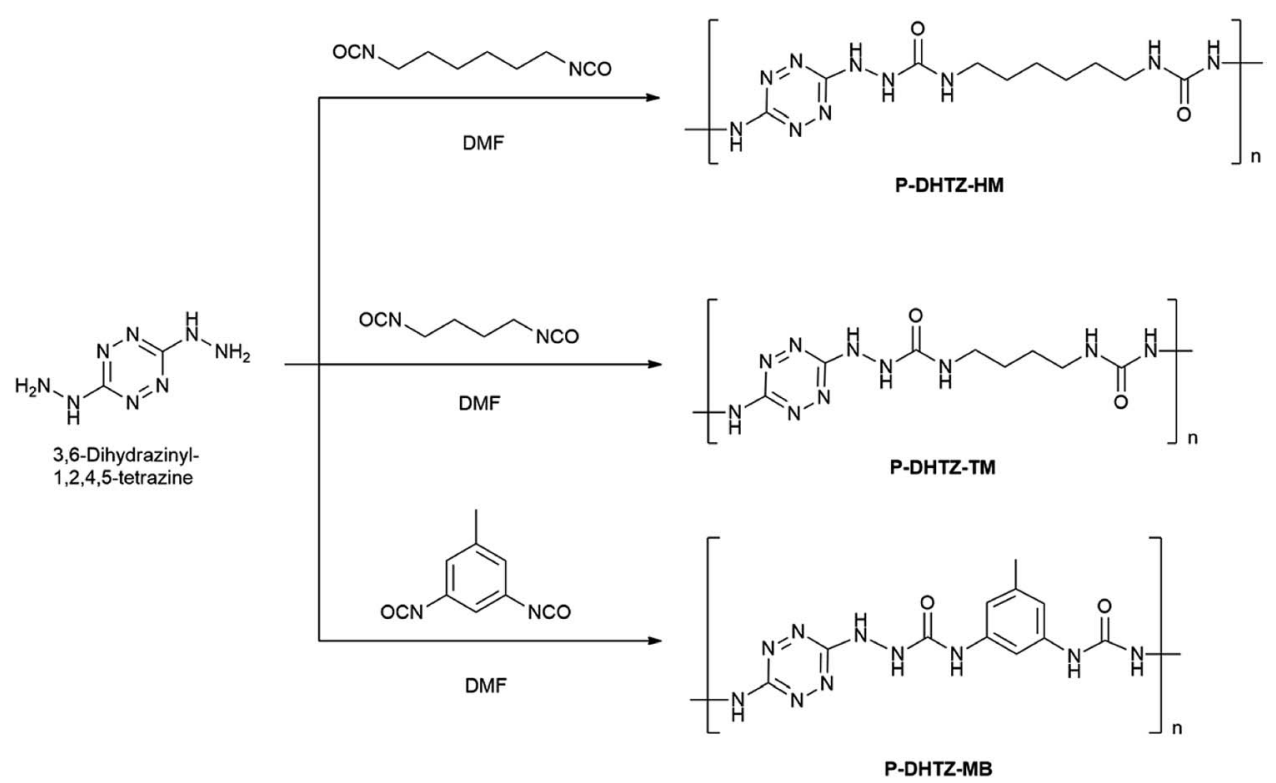

Scheme 2 Synthetic routes for P-DHTZ-HM, P-DHTZ-TM and P-DHTZ-MB polymers. 


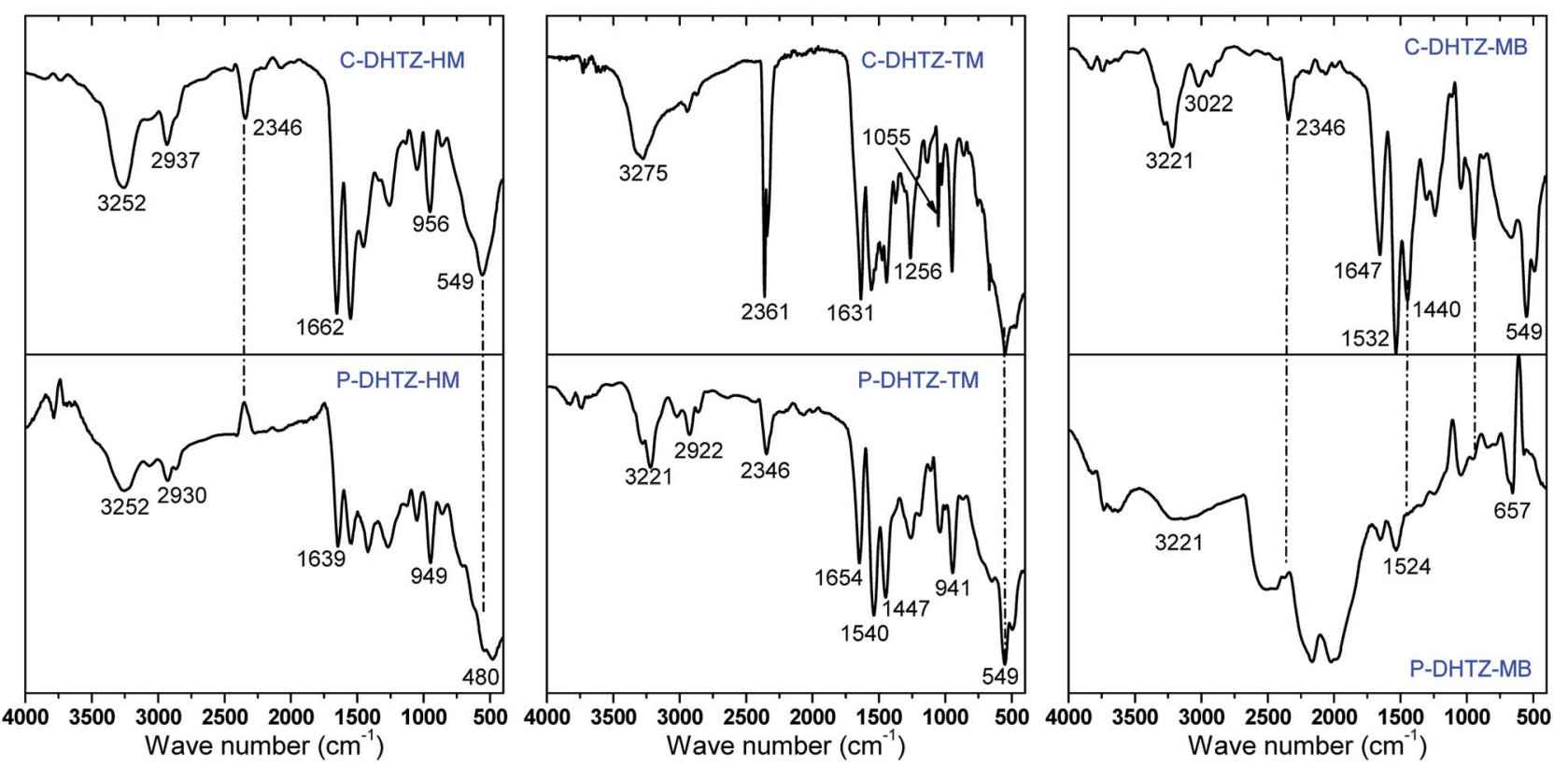

Fig. 1 Comparison of normalized FTIR spectra of DHTz-based macromolecules.

Table 1 Peak identification in FTIR spectra for DHTz-based macrocycles and polymers

\begin{tabular}{|c|c|c|c|c|c|c|}
\hline Samples & \multicolumn{6}{|c|}{ FTIR absorption peak (in $\mathrm{cm}^{-1}$ ) } \\
\hline P-DHTZ-HM & 3252 & 2930 & - & 1639 & 1417 & 480 \\
\hline C-DHTZ-TM & 3275 & 2938 & 2361 & 1631 & 1440 & 549 \\
\hline P-DHTZ-TM & 3221 & 2922 & 2346 & 1654 & 1447 & 549 \\
\hline Assignments & $\begin{array}{l}\mathrm{N}-\mathrm{H} \text { stretch in } \\
\text { amino group }\end{array}$ & $\begin{array}{l}\mathrm{C}-\mathrm{H} \text { stretch of } \\
\text { alkane chains }\end{array}$ & $\begin{array}{l}\text { Stretching or bending } \\
\text { of the entire ring }\end{array}$ & $\begin{array}{l}\mathrm{N}-\mathrm{H} \text { bending of amine } \\
\text { groups and urea's } \\
\text { carbonyl groups }\end{array}$ & $\begin{array}{l}\text { Bending of } \mathrm{C}-\mathrm{H} \\
\text { in alkane }\end{array}$ & $\begin{array}{l}\mathrm{N}-\mathrm{N} \text { bond stretching } \\
\text { in tetrazine groups }\end{array}$ \\
\hline
\end{tabular}

entrapment of DMF and water. The amount of the macrocycleentrapped DMF was in the range of $16.0 \%$ to $25.2 \%$, while the entrapped-water content reached up to $4.4 \%$ (Table 2). The latter analyses were found to be in a good agreement with the ${ }^{1} \mathrm{H}$ NMR (in DMSO- $\mathrm{d}_{6}$ ) results for the macrocycles.

\subsection{Morphologies of the macromolecules and corresponding gels}

2.3.1 Optical microscopy analysis. The obtained DHTZbased macrocycles had an appearance of amorphous dark red powders, while the polymers had brown-red colour and morphologies of films or solid blocks (Fig. 2). Based on elemental analyses of these materials, it was clear that they could possess a very high affinity to DMF and possibly function as gelators of DMF. The gelator molecules typically form multiple non-covalent interactions between themselves, which occurs in an organized manner in the gelated solvent. Indeed, upon mixing of our macrocycles with DMF or DMSO, stable sticky gels were obtained. These gels were characterized by SEM and optical microscopy (Fig. 2 and 3). Optical images of the obtained gelatinous thin films showed homogeneity of these materials, proving efficiency of macrocycles as gelators of DMF. Optimum gels formation, on a basis of DMF or DMSO, was obtained when $100 \mathrm{mg}$ of macrocycle per $1 \mathrm{ml}$ of solvent were used. Non-polymeric organic gelators are typically derived from various hydrocarbons, fatty acids, saccharides, steroids, amides, amino acids, ureas, metal complexes and dendrimers. Many urea derivatives are well known for their gelation ability in organic solvent, some of which may form a three-dimensional gel network through hydrogen bonds between the urea moieties in hexadecane, $p$-xylene, tetralin, cyclohexanone and $n$-butyl acetate..$^{32,33}$ Multiple urea functional groups in our macrocycles could be responsible for the gels formation in such highly-polar solvents as DMF and DMSO. We should mention that preparation of new energetic gels, based on our macrocycles and energetic liquids is currently in progress in our labs. 
Table 2 Elemental analysis of DHTz-based macrocycles and polymers

\begin{tabular}{|c|c|c|c|c|c|c|c|}
\hline \multirow[b]{2}{*}{ Compounds } & \multicolumn{3}{|c|}{ Calculated (\%) } & \multicolumn{3}{|c|}{ Found (\%) } & \multirow[b]{2}{*}{ Formula } \\
\hline & $\mathrm{N}$ & $\mathrm{C}$ & $\mathrm{H}$ & $\mathrm{N}$ & $\mathrm{C}$ & $\mathrm{H}$ & \\
\hline C-DHTZ-HM & 45.27 & 38.70 & 5.85 & & & & $\mathrm{C}_{20} \mathrm{H}_{36} \mathrm{~N}_{20} \mathrm{O}_{4}$ \\
\hline Containing $\mathrm{DMF} / \mathrm{H}_{2} \mathrm{O}(25.18 \% / 2.06 \%)$ & 42.40 & 39.82 & 6.25 & 42.27 & 39.90 & 6.01 & $\mathrm{C}_{23} \mathrm{H}_{43} \mathrm{~N}_{21} \mathrm{O}_{5}$ \\
\hline C-DHTZ-TM & 49.62 & 34.04 & 5.00 & & & & $\mathrm{C}_{16} \mathrm{H}_{28} \mathrm{~N}_{20} \mathrm{O}_{4}$ \\
\hline Containing $\mathrm{DMF} / \mathrm{H}_{2} \mathrm{O}(16.03 \% / 1.32 \%)$ & 44.08 & 36.04 & 5.83 & 43.68 & 36.36 & 5.63 & $\mathrm{C}_{41} \mathrm{H}_{79} \mathrm{~N}_{43} \mathrm{O}_{12}$ \\
\hline C-DHTZ-MB & 44.29 & 41.77 & 3.82 & & & & $\mathrm{C}_{22} \mathrm{H}_{24} \mathrm{~N}_{20} \mathrm{O}_{4}$ \\
\hline Containing $\mathrm{DMF} / \mathrm{H}_{2} \mathrm{O}(17.94 \% / 4.42 \%)$ & 37.82 & 41.28 & 5.20 & 37.53 & 41.41 & 5.16 & $\mathrm{C}_{28} \mathrm{H}_{42} \mathrm{~N}_{22} \mathrm{O}_{8}$ \\
\hline P-DHTZ-HM & 45.10 & 38.67 & 5.92 & & & & $\mathrm{C}_{80} \mathrm{H}_{146} \mathrm{~N}_{80} \mathrm{O}_{16}$ \\
\hline Containing $\mathrm{DMF} / \mathrm{H}_{2} \mathrm{O}(16.47 \% / 3.48 \%)$ & 39.28 & 39.10 & 6.66 & 39.07 & 39.21 & 6.05 & $\mathrm{C}_{101} \mathrm{H}_{207} \mathrm{~N}_{87} \mathrm{O}_{30}$ \\
\hline P-DHTZ-TM & 49.62 & 34.04 & 5.00 & & & & $\mathrm{C}_{96} \mathrm{H}_{168} \mathrm{~N}_{120} \mathrm{O}_{24}$ \\
\hline Containing $\mathrm{DMF} / \mathrm{H}_{2} \mathrm{O}(30.71 \% / 1.44 \%)$ & 39.55 & 38.24 & 6.52 & 39.93 & 38.67 & 6.27 & $\mathrm{C}_{159} \mathrm{H}_{323} \mathrm{~N}_{141} \mathrm{O}_{49}$ \\
\hline P-DHTZ-MB & 44.29 & 41.77 & 3.82 & & & & $\mathrm{C}_{33} \mathrm{H}_{37} \mathrm{~N}_{30} \mathrm{O}_{6}$ \\
\hline Containing DMF (10.53\%) & 37.04 & 42.80 & 5.45 & 36.82 & 43.04 & 5.27 & $\mathrm{C}_{31} \mathrm{H}_{47} \mathrm{~N}_{23} \mathrm{O}_{8}$ \\
\hline
\end{tabular}
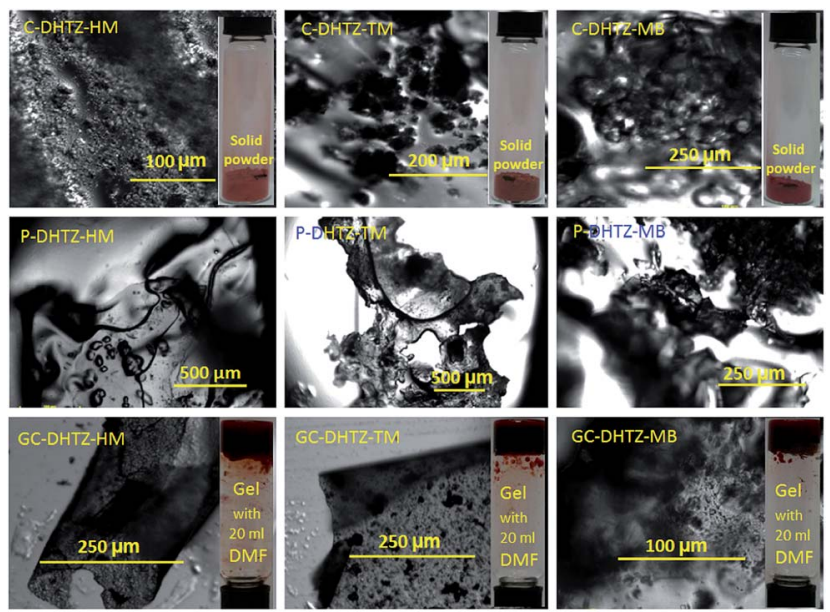

Fig. 2 Pictures and optical microscopy images of DHTZ-HM, DHTZTM, DHTZ-MB macrocycles and polymers: (top row) pure macrocycles; (middle row) polymers; (bottom row) DMF-based gels of macrocycles (images of inverted vials with gels are included).

2.3.2 SEM/EDS analysis. The morphologies and elements distribution in the macromolecules in cyclic, polymeric and gel forms were studied by SEM/EDS (energy-dispersive X-ray spectroscopy). The typical obtained SEM images are shown in Fig. 3 (corresponding EDS elemental analysis is presented in Table S3, $\mathrm{ESI} \dagger)$.

Examination of C-DHTZ-HM gel images shows this material smooth surface (Fig. 3g). In contrast, C-DHTZ-TM gel shows a self-assembly of the macrocycles in the matrix, creating bubbles on its surface (Fig. 3h). C-DHTZ-MB gel has also a rough surface, probably due to merge of gel blocks of different sizes. The structures of our gels should give them characteristic rheological properties, relevant to application of these materials as propellants. The gel propellants offer particular advantages in applications where variable thrust strength and flexible operation at an extended range are needed. The separation of the fuels and the oxidants, the gel-like consistency of both substances ensure great insensitivity and safer to handle.
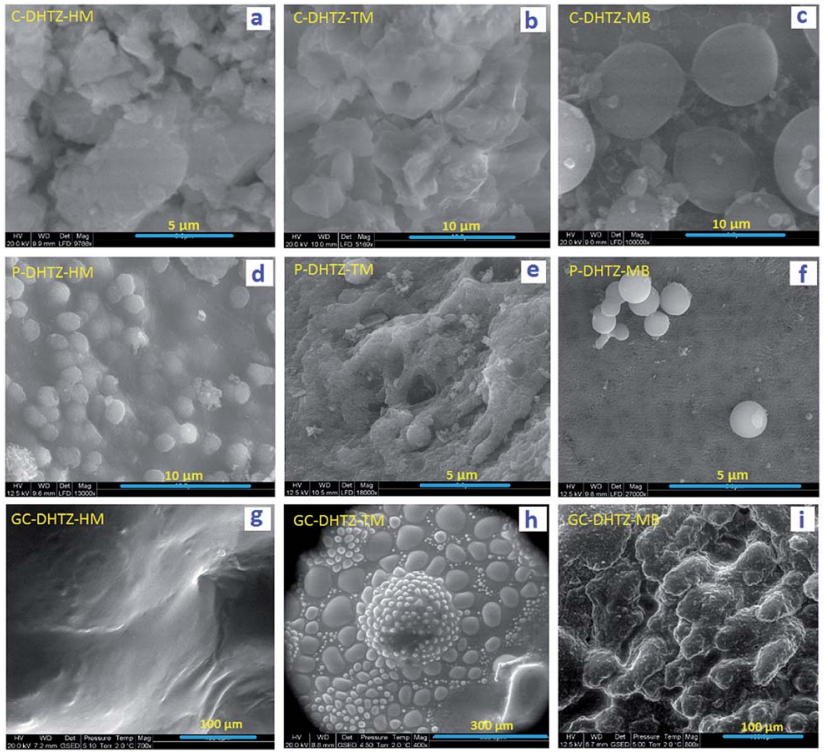

Fig. 3 SEM images of DHTZ-HM, DHTZ-TM, DHTZ-MB in their cyclic, polymeric and DMF-gel forms: (a) irregularly-shaped C-DHTZ-HM, the EDS spectrum of the imaged area indicates mainly presence of $C$, $\mathrm{N}$ and $\mathrm{O}$, with $\mathrm{N}$ content of 39\% (Table S3 $\mathrm{T}$ ); (b) C-DHTZ-TM, with N content of $47 \%$; (c) regularly-shaped C-DHTZ-MB microspheres with diameter of $8-10 \mu \mathrm{m}$ and $\mathrm{N}$ content of $32 \%$; (d) P-DHTZ-HM small particles $(1-2 \mu \mathrm{m})$ embedded into a matrix of a polymer, with $\mathrm{N}$ content of 38\%; (e) a irregularly-shaped P-DHTZ-TM polymer, with $\mathrm{N}$ content of $28 \%$; (f) P-DHTZ-MB polymer matrix (having a flat surface) decorated with several attached micro-spheres, most probably comprised of C-DHTZ-MB macrocycles (for comparison, see image $\mathrm{C}$ of this figure); (g) uniform gel of C-DHTZ-HM with DMF; (h) matrix of C-DHTZ-TM gel in DMF, embedded with bubbles or particles of the same macrocycle; (i) C-DHTZ-MB gel in DMF with rough surface, probably indicating a complex structure.

\subsection{Thermal behaviour and performances of macromolecules}

2.4.1 Measurements of heat flow properties. The heat flow DSC curves of studied materials were recorded at the heating rate of $5{ }^{\circ} \mathrm{C} \min ^{-1}$ (Fig. 4 and 5). The $\mathrm{N}_{2}$ purge gas with a flow rate of $50 \mathrm{ml} \mathrm{min}{ }^{-1}$ was used. The typical sample mass was in 

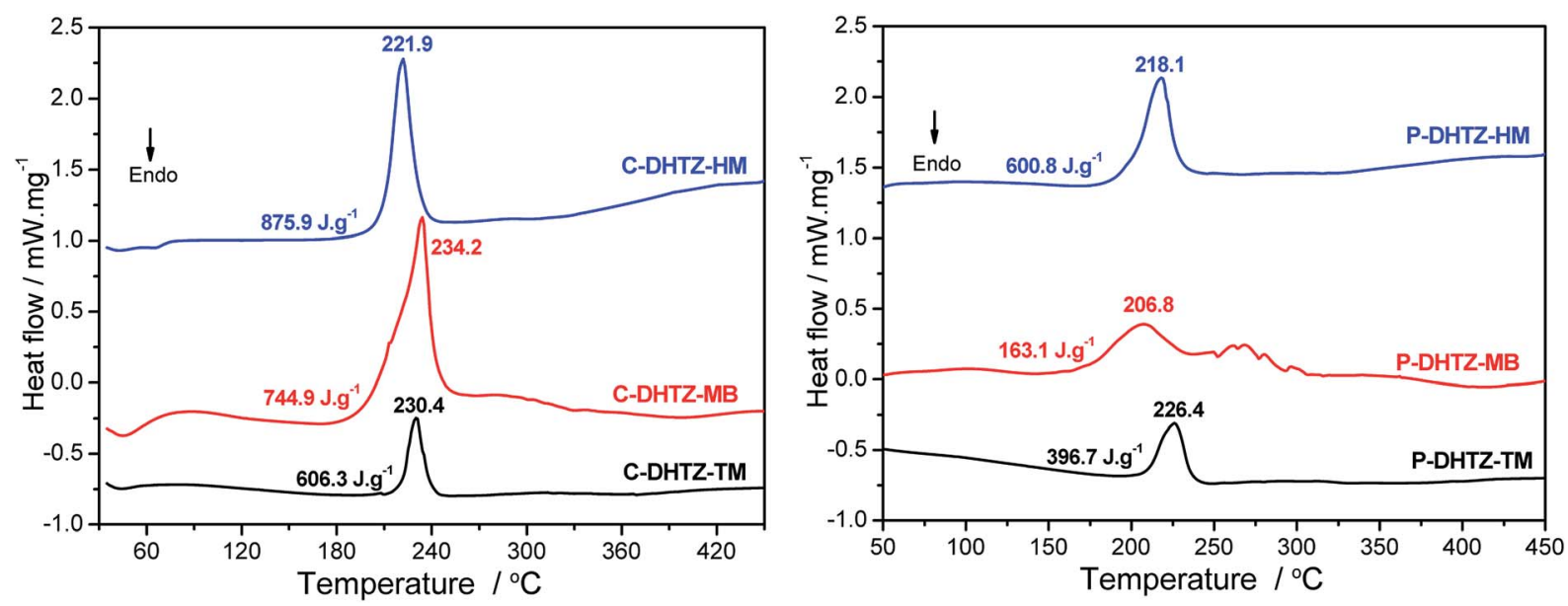

Fig. 4 DSC thermograms of DHTz-based macromolecules (heating rate of $5.0^{\circ} \mathrm{C} \mathrm{min}^{-1}$ ).

the range of 500-600 $\mu \mathrm{g}$. We found that all DHTz-based macrocycles exhibit weak and broad endothermic peaks in a range of $35-78{ }^{\circ} \mathrm{C}$. These peaks could be attributed to torsion and relaxation of macrocycle chains. The endothermic step in all macrocycles was typically followed by a single exothermic decomposition peak in the range of $220-235^{\circ} \mathrm{C}$. In contrast, the decomposition of DHTz-based polymers took place $3-10{ }^{\circ} \mathrm{C}$ earlier than their corresponding cyclic analogues. C-DHTZ-MB tolyl-based macrocycle exhibited the best thermal stability, with a shoulder peak after the main decomposition process, while among the polymers, P-DHTZ-TM (with the shortest alkyl chain) has the highest thermal stability. A quantitative comparison of the detailed heat flow parameters are shown in Table 3.

As could be seen in the Table 3, the abovementioned torsion and relaxation processes are covered by energy consumption of 73.1, 84.8 and $125.6 \mathrm{~J} \mathrm{~g}^{-1}$ for C-DHTZ-TM, C-DHTZ-MB and CDHTZ-HM, respectively. It seems that macrocycles with larger ring size $\left(-\mathrm{C}_{6} \mathrm{H}_{12}-\right)$ could undergo easier torsion and relaxation and therefore would absorb more energy for these processes. This result indicates that the cycle with larger size has lower

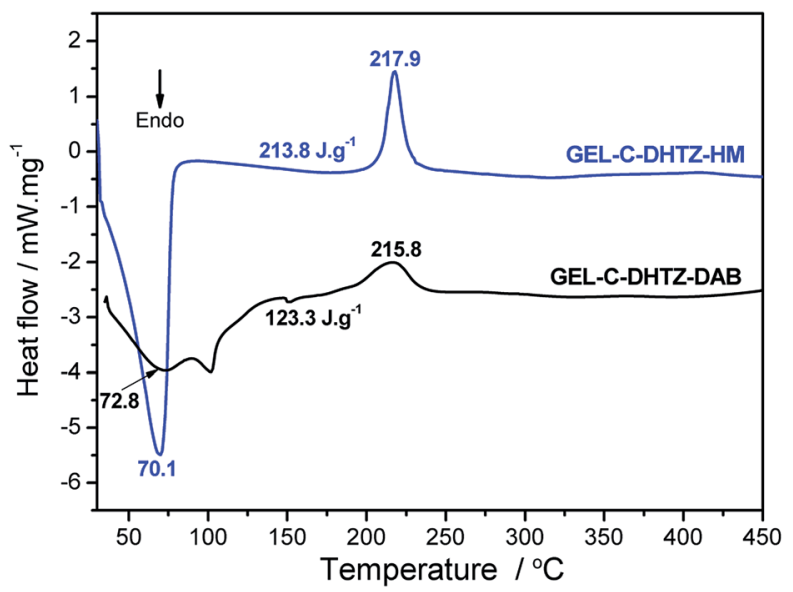

Fig. 5 DSC thermograms of DMF gels containing DHTz macrocycles (heating rate, $5.0^{\circ} \mathrm{C} \mathrm{min}^{-1}$ ). thermal stability, which is the case for C-DHTZ-HM, with the onset temperature of $209.9{ }^{\circ} \mathrm{C}$. The other two macrocycles have comparable thermal stability with high explosive $\varepsilon$ 2,4,6,8,10,12-hexanitro-2,4,6,8,10,12-hexaazaiso-wurtzitane ( $(\varepsilon-$ CL-20), which decomposes at $232-238{ }^{\circ} \mathrm{C} .{ }^{34,35}$ However, the energy content and oxygen balance of our macro-cycles are inferior to CL-20, as they have smaller heat of decomposition under oxygen lean environment.

In the case of DHTz-based polymers, it was found that alkane chain-containing polymers were generally more stable than the polymers containing tolyl building block, which starts to decompose at $178.5^{\circ} \mathrm{C}$. Importantly to note that our energetic polymers are more stable than GAP due to inclusion of more stable energetic groups and incorporation of these groups into the backbone. It has been shown that the initial thermal decomposition of GAP can begin even at $70{ }^{\circ} \mathrm{C}$, producing low molecular weight gaseous products. ${ }^{36,37}$ Although the stability of GAP could be improved by copolymerization it with BAMO. The resulted copolymer also shows a broad endothermic melting peak at $66^{\circ} \mathrm{C}$, with an enthalpy of $10 \mathrm{~J} \mathrm{~g}^{-1}$, strongly indicating that the copolymerization may induce formation crystalline domains inside otherwise amorphous GAP polymer. ${ }^{38}$ The GAP/ BAMO copolymer also shows a broad exothermic shoulder peak at $298^{\circ} \mathrm{C}$, after the main exothermic decomposition (at $228^{\circ} \mathrm{C}$ ). Our DHTz-based polymers have almost the same stability as the GAP/BAMO copolymer, especially the P-DHTZ-HM, which could be a good candidate for energetic binders of propellants and explosives. Moreover, in comparison to the P-DHTZ-HM, pol$y($ NIMMO) and poly(GLYN) exhibit much lower thermal stability with relatively fast degradation below $190{ }^{\circ} \mathrm{C}$.

The decomposition reaction of the energetic groups in GAP polymer are $-\mathrm{N}_{3}$ groups, ${ }^{39}$ while in case of poly(NIMMO) and poly(GLYN) they are $-\mathrm{ONO}_{2} \cdot{ }^{40}$ The corresponding energies of decomposition of the $-\mathrm{N}_{3}$ and $-\mathrm{ONO}_{2}$ groups are 685 and $167 \mathrm{~kJ}$ mol $^{-1}$, respectively. ${ }^{40}$ Therefore, the scission of the $-\mathrm{N}_{3}$ group results in more energy release than in the decomposition of $-\mathrm{ONO}_{2}$, which may indicate that the presence of $-\mathrm{N}_{3}$ groups leads to formation of less energetic materials than analogous 
Table 3 DSC Parameters of DHTz-based macromolecules ${ }^{a}$

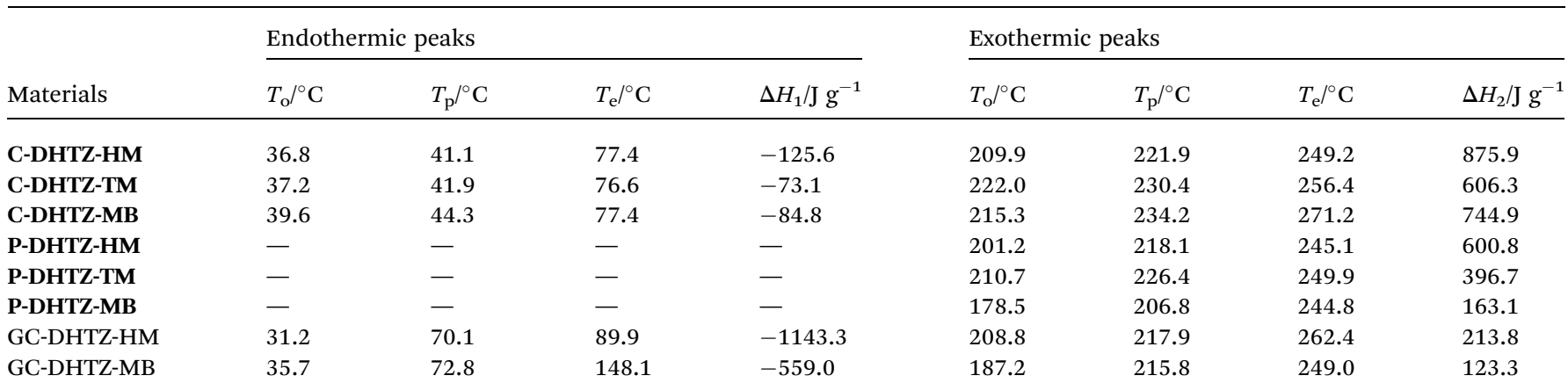

${ }^{a}$ Note: $T_{\mathrm{o}}$, onset temperature of the peaks; $T_{\mathrm{p}}$, peak temperature of thermal events; $T_{\mathrm{e}}$, the end temperature for heat change; $\Delta H_{1}$, heat absorption; $\Delta H_{2}$, heat release.

compounds containing the $-\mathrm{ONO}_{2}$ moiety. Since in DHTz-based macromolecules do not contain energetic side groups, their decomposition begins with degradation of their backbones, resulting in a higher thermal stability.

The thermal stability of the DMF gels containing DHTz macrocycles was evaluated by DSC and representative thermograms are shown in Fig. 5.

The obtained DMF gels have much lower thermal stability than corresponding solid-state macrocycles, most probably due to the dispersion effect of the solvent, which affects and weakens the intermolecular binding energy between the macrocycle molecules. It is also the case for many other crystalline energetic materials that decompose in solution, where the stabilization effect of the crystal lattice is diminished. ${ }^{41-44}$ The gel of C-DHTZ-HM shows a very broad endothermic peak (with heat absorption of $1143.3 \mathrm{~J} \mathrm{~g}^{-1}$ ), due to the dissolution of the $\mathbf{C}-$ DHTZ-HM in DMF, which is followed by evaporation of the solvent. This observation indicates that more DMF molecules are bound by C-DHTZ-HM macrocycle that has a larger ring size. It seems that the bonding effect between DMF and C-DHTZ-TM is stronger than that of C-DHTZ-HM, resulting in an endothermic shoulder peak at higher temperature (Fig. 5).

2.4.2 Physical properties and performances. The experimentally determined constant-volume heat of combustion $\left(\Delta H_{\mathrm{c}}\right)$ was measured by using oxygen bomb calorimetry. The $\Delta H_{\mathrm{c}}$ values were corrected by considering the nitric acid formation during combustion of these nitrogen-rich energetic macromolecules. The corresponding heat of formation $\left(\Delta H_{\mathrm{f}}\right)$ was calculated based on the experimental $\Delta H_{\mathrm{c}}$, when the effects of solvents were excluded. The combustion is an exothermic reaction and, consequently, $\Delta H_{\mathrm{c}}$ values obtained are negative. These thermodynamic data, together with other physical properties, including detonation and combustion parameters, predicted by EXPLO-5 code, are listed in Table 4 . The calculated $\Delta H_{\mathrm{f}}$ for C-DHTZ-HM, C-DHTZ-TM and C-DHTZ-MB macrocycles are $-2.39 \mathrm{~kJ} \mathrm{~g}^{-1}$, $-0.63 \mathrm{~kJ} \mathrm{~g}^{-1}$ and $-1.54 \mathrm{~kJ} \mathrm{~g}^{-1}$, respectively. The P-DHTZ-HM and P-DHTZ-MB polymers exhibited larger $\Delta H_{\mathrm{f}}$ than their cyclic counterparts. In principle, all macrocycles should have thermodynamically stable structures under ambient conditions. The $\Delta H_{\mathrm{c}}$ of P-DHTZ-HM polymer was found to be $-20.76 \mathrm{~kJ} \mathrm{~g}^{-1}$, which is slightly larger than that of GAP.

Table 4 Explosive properties and performance of DHTz-based energetic macromolecules and reference compounds ${ }^{a}$

\begin{tabular}{|c|c|c|c|c|c|c|c|c|c|}
\hline & C-DHTZ-HM & C-DHTZ-TM & C-DHTZ-MB & P-DHTZ-HM & P-DHTZ-TM & P-DHTZ-MB & $\mathrm{RDX}$ & $\mathrm{GAP}_{2900}$ & $\operatorname{poly}(\mathrm{GLYN})$ \\
\hline Formula & $\mathrm{C}_{20} \mathrm{H}_{36} \mathrm{~N}_{20} \mathrm{O}_{4}$ & $\mathrm{C}_{16} \mathrm{H}_{28} \mathrm{~N}_{20} \mathrm{O}_{4}$ & $\mathrm{C}_{22} \mathrm{H}_{24} \mathrm{~N}_{20} \mathrm{O}_{4}$ & $\mathrm{C}_{11} \mathrm{H}_{20} \mathrm{~N}_{10} \mathrm{O}_{2}$ & $\mathrm{C}_{9} \mathrm{H}_{16} \mathrm{~N}_{10} \mathrm{O}_{2}$ & $\mathrm{C}_{11} \mathrm{H}_{12} \mathrm{~N}_{10} \mathrm{O}_{2}$ & $\mathrm{C}_{3} \mathrm{H}_{6} \mathrm{~N}_{6} \mathrm{O}_{6}$ & $\mathrm{C}_{3} \mathrm{H}_{5} \mathrm{~N}_{3} \mathrm{O}$ & $\mathrm{C}_{3} \mathrm{H}_{5} \mathrm{NO}_{4}$ \\
\hline$M_{\mathrm{w}}$ & 620.6 & 564.5 & 632.6 & 324.4 & 296.3 & 316.3 & 222.1 & 99.1 & 119.0 \\
\hline N\% & 45.1 & 49.6 & 44.3 & 43.2 & 47.3 & 44.3 & 37.8 & 42.41 & 9.3 \\
\hline$T_{\mathrm{p}}$ & 221.9 & 230.4 & 234.2 & 218.1 & 226.4 & 206.8 & 241.3 & 200.1 & 204.3 \\
\hline$\Delta H_{\mathrm{c}}(\mathrm{sol})$ & -19.80 & -18.59 & -17.61 & -20.26 & -17.71 & -19.15 & - & - & - \\
\hline$\rho$ & 1.58 & 1.82 & 1.50 & 1.27 & 1.34 & 1.17 & 1.82 & 1.30 & 1.46 \\
\hline VoD & 4195 & 7626 & 4399 & 4611 & - & 4831 & 8760 & 5041 & 6755 \\
\hline Gas vol. & 745 & 752 & 649 & 751 & - & 708 & 836 & 739 & 838 \\
\hline$I_{\mathrm{sp}}$ & 169 & 191 & 171 & 182 & 117 & 199 & 270 & 217 & 176 \\
\hline
\end{tabular}

${ }^{a}$ Notes: $M_{\mathrm{w}}$, molecular weight, in $\mathrm{g} \mathrm{mol}^{-1} ; \Delta H_{\mathrm{c}}(\mathrm{sol})$, measured heat of combustion for as-prepared macromolecules, in kJ g ${ }^{-1} ; \Delta H_{\mathrm{c}}$, calculated heat

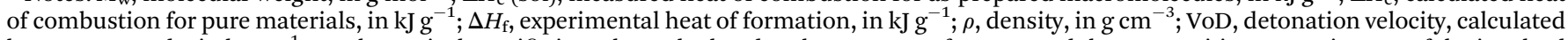
by EXPLO-5 code, in $\mathrm{km} \mathrm{s}^{-1} ; I_{\mathrm{sp}}$, theoretical specific impulse, calculated at the pressure of $7 \mathrm{MPa}$, and the compositions contain $40 \%$ of the involved compound and $60 \%$ of ammonia dinitramide (ADN), in $\mathrm{s}^{-1}$; gas vol., calculated volume of detonation gas products (EXPLO-5) at standard conditions, in $\mathrm{dm}^{3} \mathrm{~kg}^{-1 n}$ ( $n=1$ for cycles). 
Since we could not establish exactly the average $M_{\mathrm{w}}$ of our DHTz-based polymer, we used $M_{\mathrm{w}}$ and $\Delta H_{\mathrm{f}}$ of a single repeating unit of each polymer for EXPLO-5 calculations. It was previously reported that changes in the $M_{\mathrm{w}}$ of the GAP don't affect the $\Delta H_{\mathrm{c}}$ of this polymer. ${ }^{45}$ Therefore, the $\Delta H_{\mathrm{f}}$ of our repeating units could be calculated on a basis of $\Delta H_{\mathrm{c}}$ of their polymers. We found that $\Delta H_{\mathrm{c}}$ values for all our polymers are larger that of the poly(GLYN) (Table 4). In case of nitrogen-rich polymers, containing energetic moieties in the backbone, their $\Delta H_{\mathrm{c}}$ values are practically determined by the chain structures. The combustion of a common energetic polymer usually starts with the degradation of its energetic groups.

We found that C-DHTZ-TM macrocycle has a higher VoD (7626 $\mathrm{m} \mathrm{s}^{-1}$ ) than TNT, while P-DHTZ-HM polymer has a larger detonation gas production than GAP, due to higher nitrogen content. Safety measurements of the most energetic macrocycle C-DHTZ-TM showed high insensitivity to impact (10 kg hammer, $H_{50}=64.2 \mathrm{~J}$ ) and friction $(36 \mathrm{~kg},>352.8 \mathrm{~N})$. The VoDs of all our polymers are less than $5 \mathrm{~km} \mathrm{~s}^{-1}$, which is slightly lower than that of the GAP. However, the ADN based compositions, bonded by $60 \mathrm{wt} \%$ of P-DHTZ-HM or P-DHTZ-MB polymers, have higher theoretical $I_{\mathrm{sp}}$ than ADN-bonded by poly(GLYN).

\section{Experimental}

\subsection{General}

Reagents and solvents used in this work were of high grades and were used as supplied. ${ }^{1} \mathrm{H}$ and ${ }^{13} \mathrm{C}$ NMR spectra were recorded on a $400 \mathrm{MHz}$ NMR spectrometer. Infrared spectra were recorded on a FTIR spectrometer equipped with a diamond ATR unit. Mass spectra were recorded on a high-resolution ESI-Q-ToF machine. Elemental analyses (CHN) were performed at the service facility of the Hebrew University. Decomposition points were determined by differential scanning calorimetry (DSC). Measurements were performed at a heating rate of $5{ }^{\circ} \mathrm{C} \mathrm{min}-1$ in closed aluminium sample pans with a small hole in the lid,

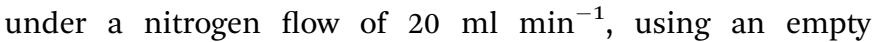
aluminium sample pan as a reference. Melting points were measured on a melting point apparatus in open glass capillaries. The XRD spectra were obtained using $\mathrm{Cu} \mathrm{K} \alpha$ at voltage of $30 \mathrm{kV}$, where the scan ranges is $5-70 \mathrm{deg}$. at a speed of $0.5 \mathrm{deg}$. $\min ^{-1}$. The experimental densities were obtained by pycnometry measurements at ambient temperature.

\subsection{Synthesis of C-DHTZ-HM}

To a solution of DHTz (145 mg, $0.1 \mathrm{mmol})$ in a mixture of DMF/ THF (30 ml, $1: 1 \mathrm{v} / \mathrm{v}$ ) 1,6-hexamethylene diiso-cyanate (168 mg, $0.1 \mathrm{mmol}$ ) was added and the resulted solution was stirred at room temperature for overnight. Formed precipitate was filtered, washed with THF $(3 \times 20 \mathrm{ml})$, water $(3 \times 50 \mathrm{ml})$ and vacuum dried. A crude solid material was re-dissolved in DMF $(20 \mathrm{ml})$ at $100{ }^{\circ} \mathrm{C}$ and water $(5 \mathrm{ml})$ was added dropwise. Upon cooling to room temperature a pure C-DHTZ-HM precipitated. It was filtered out, washed with THF $(3 \times 20 \mathrm{ml})$ and vacuum dried. Yield: $51 \% . T_{\text {decomp. }}$ (DSC) $227^{\circ} \mathrm{C}$. FTIR (ATR, $\mathrm{cm}^{-1}$ ): 3264 (w), 2929 (m), 2346 (m), 1648 (m), 1533 (m), 1437 (m), 1256 (s),
1055 (m), 950 (m), 568 (s). ${ }^{1} \mathrm{H}$ NMR (400 MHz, DMSO-d $\left.{ }_{6}\right) \delta: 0.33$ $\left(\mathrm{s}, 8 \mathrm{H}, \mathrm{CH}_{2}\right), 0.48\left(\mathrm{~s}, 8 \mathrm{H}, \mathrm{CH}_{2}\right), 2.10\left(\mathrm{~s}, 8 \mathrm{H}, \mathrm{CH}_{2}\right), 5.68(\mathrm{bs}, 4 \mathrm{H}$, $\mathrm{NH}), 7.05(\mathrm{~s}, 4 \mathrm{H}, \mathrm{NH}), 8.31(\mathrm{~s}, 4 \mathrm{H}, \mathrm{NH}) ;{ }^{13} \mathrm{C} \mathrm{NMR}(100 \mathrm{MHz}$, DMSO-d $\left.{ }_{6}\right) \delta: 26.5\left(\mathrm{CH}_{2}\right), 30.3\left(\mathrm{CH}_{2}\right), 39.6\left(\mathrm{CH}_{2}\right), 159.0(\mathrm{C}=\mathrm{O})$, $163.4(\mathrm{C}=\mathrm{N})$. HRMS (ESI-): $m / z=619.3146\left[\mathrm{M}-\mathrm{H}^{-}\right]$.

\subsection{Synthesis of C-DHTZ-TM}

To a solution of DHTz (145 mg, $0.1 \mathrm{mmol})$ in DMF (30 ml) 1,4diisocyanatobutane $(145 \mathrm{mg}, 0.1 \mathrm{mmol}$ ) was added and the resulted solution was stirred at room temperature for overnight. Formed precipitate was filtered, washed with THF $(3 \times 20 \mathrm{ml})$, water $(3 \times 50 \mathrm{ml})$ and vacuum dried. A crude solid material was redissolved in DMF $(10 \mathrm{ml})$ at $90{ }^{\circ} \mathrm{C}$ and water $(3 \mathrm{ml})$ was added dropwise. Upon cooling to room temperature a pure C-DHTZ-TM precipitated. It was filtered out, washed with THF $(3 \times 20 \mathrm{ml})$ and

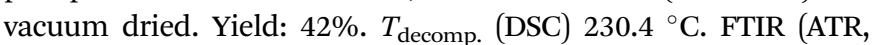
$\mathrm{cm}^{-1}$ ): 3271 (w), 2351 (s), 2321 (s), 1625 (m), 1566 (m), 1439 (m), 1244 (m), 1037 (s), 959 (m), 567 (m). ${ }^{1} \mathrm{H}$ NMR (400 MHz, DMSO-d ${ }_{6}$ ) $\delta: 1.30$ (bs, 8H, $\mathrm{CH}_{2}$ ), 2.87 (bs, 8H, $\mathrm{CH}_{2}$ ), 6.55 (bs, 4H, NH), 7.93 (s, $4 \mathrm{H}, \mathrm{NH}), 9.20(\mathrm{~s}, 4 \mathrm{H}, \mathrm{NH}) .{ }^{13} \mathrm{C} \mathrm{NMR}\left(100 \mathrm{MHz}, \mathrm{DMSO}_{6}\right) \delta: 26.2$ $\left(\mathrm{CH}_{2}\right), 37.9\left(\mathrm{CH}_{2}\right), 151.6(\mathrm{C}=\mathrm{O}), 161.9(\mathrm{C}=\mathrm{N}) .{ }^{13} \mathrm{C}$ DEPT135 (100 MHz, DMSO-d $\left.{ }_{6}\right) \delta: 26.2\left(\mathrm{CH}_{2}\right), 37.9\left(\mathrm{CH}_{2}\right)$. HRMS (ESI-): $m / z=$ $587.2502\left[\mathrm{M}-\mathrm{H}^{-}\right]$.

\subsection{Synthesis of C-DHTZ-MB}

To a solution of DHTz (145 mg, $0.1 \mathrm{mmol}$ ) in a mixture of DMF/ THF (30 ml, $1: 1 \mathrm{v} / \mathrm{v}$ ) toluene-2,4-diisocyanate (175 mg, 0.1 mmol) was added and the resulted solution was stirred at room temperature for overnight. Formed precipitate was filtered, washed with THF $(3 \times 20 \mathrm{ml})$, water $(3 \times 50 \mathrm{ml})$ and vacuum dried. A crude solid material was re-dissolved in DMF $(20 \mathrm{ml})$ at $60{ }^{\circ} \mathrm{C}$ and water $(20 \mathrm{ml})$ was added dropwise. Upon slow cooling to room temperature a pure C-DHTZ-MB precipitated. It was filtered out, washed with THF $(3 \times 20 \mathrm{ml})$ and vacuum dried. Yield: $57 \%$. $T_{\text {decomp. }}(\mathrm{DSC})$ $234.2{ }^{\circ}$ C. FTIR (ATR, $\mathrm{cm}^{-1}$ ): $3738(\mathrm{~m}), 3630(\mathrm{~m}), 3178(\mathrm{w}), 2521(\mathrm{w})$, 2171 (m), 2009 (m), 1538 (m), 1045 (m), 640 (s). (400 MHz, DMSO$\left.\mathrm{d}_{6}\right) \delta: 2.10\left(\mathrm{~s}, 6 \mathrm{H}, \mathrm{CH}_{3}\right), 7.04(\mathrm{~s}, 4 \mathrm{H}, \mathrm{NH}), 7.66-8.82(\mathrm{~m}, 10 \mathrm{H}, 4 \mathrm{NH}+$ 6 aromatics), $9.43(\mathrm{~s}, 4 \mathrm{H}, \mathrm{NH}) .{ }^{13} \mathrm{C}$ NMR (100 MHz, DMSO-d 6 ) $\delta$ : $17.2\left(\mathrm{CH}_{3}\right), 110.3(\mathrm{CH}$, aromatic), $126.1(\mathrm{CH}$, aromatic), $129.6(\mathrm{C}-$ $\mathrm{NH}$, aromatic), $137.8\left(\mathrm{C}-\mathrm{CH}_{3}\right.$, aromatic), $155.8(\mathrm{C}=\mathrm{O}), 162.0(\mathrm{~N}-\mathrm{C}-$ $\mathrm{N}$ tetrazine). HRMS (ESI-): $m / z=631.2205\left[\mathrm{M}-\mathrm{H}^{-}\right]$.

\subsection{Synthesis of P-DHTZ-HM}

To a dispersion of DHTz (145 mg, $0.1 \mathrm{mmol}$ ) in DMF (1 ml) 1,6hexamethylene diisocyanate $(168 \mathrm{mg}, 0.1 \mathrm{mmol})$ was added and the resulted solution was stirred at room temperature for overnight. Formed rubber-like red-orange solid was filtered, washed with DMF $(3 \times 20 \mathrm{ml})$, water $(5 \times 100 \mathrm{ml})$ and extensively vacuum dried to give P-DHTZ-HM polymer. $T_{\text {decomp. }} 218.1$ ${ }^{\circ}$ C. FTIR (ATR, cm ${ }^{-1}$ ): 3708 (m), 3243 (w), 2937 (m), 1647 (s), 1551 (s), 1413 (m), 1262 (m), 1051 (m), 943 (s), 479 (w).

\subsection{Synthesis of P-DHTZ-TM}

To a dispersion of DHTz (145 mg, $0.1 \mathrm{mmol}$ ) in DMF (1 ml) 1,4biiso-cyanatobutane $(145 \mathrm{mg}, 0.1 \mathrm{mmol})$ was added and the 
resulted solution was stirred at room temperature for overnight. Formed rubber-like red-orange solid was filtered, washed with DMF $(3 \times 20 \mathrm{ml})$, water $(5 \times 100 \mathrm{ml})$ and extensively vacuum dried to give P-DHTZ-TM polymer. $T_{\text {decomp. }}(\mathrm{DSC}) 226.4^{\circ} \mathrm{C}$. FTIR (ATR, $\mathrm{cm}^{-1}$ ): $3226(\mathrm{~m}), 2931$ (m), $2340(\mathrm{~m}), 1647$ (s), 1533 (s), 1448 (s), 1261 (m), $1044(\mathrm{~m}), 943(\mathrm{~s}) 544(\mathrm{~s})$.

\subsection{Synthesis of P-DHTZ-MB}

To a dispersion of DHTz (145 mg, $0.1 \mathrm{mmol})$ in DMF $(1 \mathrm{ml})$ toluene-2,4-diisocyanate ( $175 \mathrm{mg}, 0.1 \mathrm{mmol}$ ) was added and the resulted solution was stirred at room temperature for overnight. Formed rubber-like red-orange solid was filtered, washed with DMF $(3 \times 20 \mathrm{ml})$, water $(5 \times 100 \mathrm{ml})$ and extensively vacuum dried to give P-DHTZ-MB polymer. $T_{\text {decomp. }}$ (DSC) $206.8^{\circ} \mathrm{C}$. FTIR (ATR, $\mathrm{cm}^{-1}$ ): $3220(\mathrm{w}), 2340(\mathrm{~m}), 1659(\mathrm{~s}), 1520(\mathrm{~s}), 1442(\mathrm{~s}), 1316$ (m), $1238(\mathrm{~s}), 1039(\mathrm{~m}), 949(\mathrm{~s}), 666(\mathrm{w}), 551(\mathrm{~s}), 485(\mathrm{~m})$.

\section{Conclusions}

A new family of DHTz-based energetic macrocycles and polymers was prepared by using toluene-2,4-diisocyanate, 1,4-diisocyanatobutane and 1,6-diisocyanato-hexane. In order to obtain macrocycles, reactions need to be performed in DMF, under highdilution conditions. In contrast, for the corresponding energetic polymers, high concentration of the starting materials in mixtures of DMSO/THF or DMF/THF was required.

From DSC measurements, we found that the thermal stability ofDHTz-based energetic materials was higher than of the currently used energetic polymers, such as GAP, poly(NIMMO) and poly-(GLYN). Moreover, C-DHTZ-TM macrocycle has calculated VoD of $7626 \mathrm{~m} \mathrm{~s}^{-1}$ (EXPLO-5), while P-DHTZHM has calculated detonation gas production larger than GAP due to it higher nitrogen content. We also calculated that the ADN-based compositions, bonded by $60 \mathrm{wt} \%$ of P-DHTZ-HM or P-DHTZ-MB polymers exhibited higher $I_{\mathrm{sp}}$ than ADN bonded by poly(GLYN). Characterization of our new energetic macromolecules revealed the presence of significant amounts of DMF entrapped within their molecular mesh in solid state. These observations lead us to evaluate DMF-soluble cyclic macrocycles as gelators for this solvent, while the corresponding polymers were found to be practically insoluble in all tested solvents. The structures of our gels should give these materials characteristic rheological properties, relevant to their application as propellants. The gel propellants offer particular advantages in applications where variable thrust strength and flexible operation at an extended range are required. The separation of the fuels and the oxidants, the gellike consistency of both substances ensure great insensitivity and safer to handle. Therefore, we believe that our new highrich macromolecules could be useful as promising energetic binders or gelators for various propellants.

\section{Acknowledgements}

The authors are thankful to the Momentum Foundation and Tel Aviv University for funding on this project.

\section{Notes and references}

1 B. Gaur, B. Lochab, V. Choudhary and I. K. Varma, Azido Polymers - Energetic Binders for Solid Rocket Propellants, J. Macromol. Sci., Polym. Rev., 2003, 43, 505-545.

2 Y. M. Mohan, K. M. Raju and B. Sreedhar, Synthesis and characterization of glycidyl azide polymer with enhanced azide content, Int. J. Polym. Mater. Polym. Biomater., 2006, 55, 441-455.

3 Y. M. Mohan and K. M. Raju, Synthesis and characterization of low molecular weight azido polymers as high energetic plasticizers, Int. J. Polym. Anal. Charact., 2004, 9, 289-304.

4 E. A. Murphy, T. Ntozakhe, C. J. Murphy, J. J. Fay and L. H. Sperling, Characterization of poly(3,3-bisethoxymethyl oxetane) and poly(3,3-bisazidomethyl oxetane) and their block copolymers, J. Appl. Polym. Sci., 1989, 37, 267-281.

5 G. Wang, Z. Ge and Y. Luo, Synthesis and Characterization of Poly(3-azidomethyl-3-methyl oxetane) by the Azidation of Poly(3-mesyloxymethyl-3-methyl oxetane), Propellants, Explos., Pyrotech., 2015, 40(6), 920-926, DOI: 10.1002/ prep.201500064.

6 P. F. Bunyan, An investigation of the thermal decomposition of poly(3-nitratomethyl-3-methyloxetane), Thermochim. Acta, 1992, 207, 147-159.

7 E. Kimura, Y. Oyumi, H. Kawasaki, Y. Maeda and T. Anan, Characterization of BAMO/NMMO copolymers, Propellants, Explos., Pyrotech., 1994, 19(5), 270-275.

8 W. Rodney, G. S. Alfred and S. D. Robert, Polyglycidyl nitrate plasticizers, US pat., 5380777 A, Jan 1995.

9 A. Kazemi, M. Hayaty, A. Mousaviazar, K. A. Samani and M. H. Keshavarz, The synthesis and characterization of polyvinyl nitrate as an energetic polymer and study of its thermal behavior, J. Therm. Anal. Calorim., 2015, 119, 613-618.

10 P. F. Bunyan, An investigation of the thermal decomposition of poly(3-nitratomethyl-3-methyloxetane), Thermochim. Acta, 1992, 207, 147-159.

11 K. Menke, T. Heintz, W. Schweikert, T. Keicher and H. Krause, Formulation and Properties of ADN/GAP propellants, Propellants, Explos., Pyrotech., 2009, 34, 218-230.

12 Z. Zhang, G. Wang, Z. Wang, Y. Zhang, Z. Ge and Y. Luo, Synthesis and characterization of novel energetic thermoplastic elastomers based on glycidyl azide polymer (GAP) with bonding functions, Polym. Bull., 2015, 72, 1835-1847.

13 Y. Noda, S. Ono and K. Ohno, Geometry dependence of electronic and energetic properties of one-dimensional peanut-shaped fullerene polymers, J. Phys. Chem. A, 2015, 119, 3048-3055.

14 A. M. Kawamoto, M. F. Diniz, V. L. Lourencedil'o, M. F. K. Takahashi, T. Keicher, H. Krause, K. Menke and P. B. Kempa, Synthesis and characterization of GAP/BAMO copolymers applied at high energetic composite propellants, J. Aerosp. Technol. Manage., 2010, 2, 307-322.

15 Y. M. Mohan, Y. Mani and K. M. Raju, Synthesis of azido polymers as potential energetic propellant binders, Des. Monomers Polym., 2006, 9, 201-236. 
16 A. J. Bellamy, D. S. King and P. Golding, Synthesis of energetic polymers by the introduction of energetic groups onto polymeric primary and secondary amines, Propellants, Explos., Pyrotech., 2004, 29, 166-177.

17 B. Jin, J. Shen, R. Peng, Y. Shu, B. Tan, S. Chu and H. Dong, Synthesis, characterization, thermal stability and sensitivity properties of the new energetic polymer through the azidoacetylation of poly(vinyl alcohol), Polym. Degrad. Stab., 2012, 97, 473-480.

18 V. N. Kizhnyaev, F. A. Pokatilov and L. I. Vereshchagin, Carbon chain Polymers with Oxadiazole, Triazole, and Tetrazole Cycles, Polym. Sci., Ser. C, 2008, 50, 1-21.

19 G. Clavier and P. S. Audebert, Tetrazines as building blocks for new functional molecules and molecular materials, Chem. Rev., 2010, 110, 3299-3314.

20 E. Sagot, A. le Roux, C. Soulivet, E. Pasquinet, D. Poullain, E. Girard and P. Palmas, Synthesis of linear and hyper branched tetrazine-based polyhetarylene assemblies with high nitrogen content, Tetrahedron, 2007, 63, 11189-11194.

21 C. G. Overberger and F. W. Michelotti, The Preparation of Polymers and Copolymers from Vinylpyrimidines and Triazines, J. Am. Chem. Soc., 1958, 80, 988-991.

22 G. F. L. Ehlers and J. D. Ray, Synthesis and Characterization of Poly-s-Triazinyleneimides, J. Polym. Sci., Part A: Gen. Pap., 1964, 2, 4989-5003.

23 P. D. Oja, W. Creek and E. T. Niles., High Nitrogen Polymers Prepared by Reacting Aldehydes with Aminoguanidines, Tetrazoles, or Triazoles, US Pat., 3375230, 1968.

24 T. M. Klapötke and S. M. Sproll, Investigation of NitrogenRich Energetic Polymers Based on Alkylbridged Bis-(1methyl-tetrazolylhydrazines), J. Polym. Sci., Part A: Polym. Chem., 2010, 48, 122-127.

25 F. M. Betzler, R. Boller, A. Grossmann and T. M. Klapötke, Novel insensitive energetic nitrogen-rich polymers based on tetrazoles, J. Chem. Sci., 2013, 68, 714-718.

26 E. Sagot, A. le Roux, C. Soulivet, E. Pasquinet, D. Poullain, E. Girard and P. Palmas, Synthesis of Linear and Hyperbranched Tetrazine-Based Polyhetarylenes Assemblies with High Nitrogen Content, Tetrahedron, 2007, 63, 1118911194.

27 M. Mahkam, M. Nabati, A. Latifpour and J. Aboudi, Synthesis and characterization of new nitrogen-rich polymers as candidates for energetic applications, Des. Monomers Polym., 2014, 17, 453-457.

28 Y.-H. Gong, P. Audebert, G. Clavier, F. Miomandre, J. Tang, S. Badré, R. Méallet-Renault and E. Naidus, Preparation and physicochemical studies of new multiple rings $s$ tetrazines, New J. Chem., 2008, 32, 1235-1242.

29 J. Ding, Z. Li, Z. Cui, G. P. Robertson, N. Song, X. Du and L. Scoles, The preparation of 3,6-bis(3-hexylthien-2-yl)-stetrazine and its conjugated polymers, J. Polym. Sci., Part A: Polym. Chem., 2011, 49, 3374-3386.

30 Q. Zhou, Synthesis of new tetrazines functionalized with photoactive and electroactive groups. PhD diss., École normale supérieure de Cachan-ENS Cachan, 2012.
31 G. R. Newkome, A. Nayak, F. Fronczek, T. Kawato, H. C. R. Taylor, L. Meade and W. Mattice, Synthesis and temperature-dependent conformational preferences of macrocycles containing the 2,2'-bipyridyl moiety, J. Am. Chem. Soc., 1979, 101, 4472-4477.

32 J. van Esch, S. de Feyter, R. M. Kellogg, F. D. Schryver and B. L. Feringa, Chem.-Eur. J., 1997, 3, 1238-1243.

33 F. S. Schoonbeek, J. H. van Esch, R. Hulst, R. M. Kellogg and B. L. Feringa, Chem.-Eur. J., 2000, 6, 2633-2643.

34 Q.-L. Yan, S. Zeman, R. Svoboda, A. Elbeih and J. Málek, The effect of crystal structure on the thermal initiation of CL-20 and its C4 bonded explosives (II): models for overlapped reactions and thermal stability, J. Therm. Anal. Calorim., 2013, 112, 837-849.

35 Q.-L. Yan, S. Zeman, A. Elbeih, Z.-W. Song and J. Málek, The effect of crystal structure on the thermal reactivity of CL-20 and its C4 bonded explosives (I): thermodynamic properties and decomposition kinetics, J. Therm. Anal. Calorim., 2013, 112, 823-836.

36 T.-F. Wang and S.-F. Li, The thermal decomposition and combustion of a new type high energy polymer GAP, Chin. J. Explos. Propellants, 2005, 28, 5-8.

37 O. P. Korobeinichev, L. V. Kuibida, E. N. Volkov and A. G. Shmakov, Mass spectrometric study of combustion and thermal decomposition of GAP, Combust. Flame, 2002, 129, 136-150.

38 S. Pisharath and H. G. Ang, Synthesis and thermal decomposition of GAP-Poly(BAMO) copolymer, Polym. Degrad. Stab., 2007, 92, 1365-1377.

39 N. Kubota, T. Sonobe, A. Yamamoto and H. Shimizu, Burning Rate Characteristics of GAP Propellants, J. Propul. Power, 1990, 6, 686.

40 P. F. Bunyan, B. W. Clements, A. V. Cunliffe, H. Desai and S. A. Torry, Structure of Energetic Ether Prepolymers, Insensitive Munitions and Energetic Materials Technology Symposium, 1997, Orlando, FL, USA, pp. 6-9, NDIA.

$41 \mathrm{H}$. Cheradame, J. P. Andreolety and E. Rousset, Homopolymerization of 3,3-bis(azidomethyl)oxetane and its copolymerization with 3-chloromethyl-3-(2,5,8trioxadecyl) oxetane, Makromol. Chem., 1991, 192, 901-918.

42 S. Zeman, Sensitivity of high energy compounds, in High Energy Density Materials, Series: Structure \& Bonding, ed. T. Klapoetke, Springer, New York, 2007, vol. 125, pp. 195-271.

43 S. Zeman and Z. Friedl, Relationship between electronic charges at nitrogen atoms of nitro groups and onsets of thermal decomposition of polynitro arenes, Cent. Eur. J. Energ. Mater., 2004, 1, 3-21.

44 Q.-L. Yan, S. Zeman, J. Šelešovský, R. Svoboda and A. Elbeih, Thermal behavior and decomposition kinetics of Formexbonded explosives containing different cyclic nitramines, $J$. Therm. Anal. Calorim., 2013, 111, 1419-1430.

45 E. Diaz, P. Brousseau, G. Ampleman and R. E. Prud'homme, Heats of combustion and formation of new energetic thermoplastic elastomers based on GAP, polyNIMMO and polyGLYN, Propellants, Explos., Pyrotech., 2003, 28, 101-106. 